\title{
Biostratigraphische Begriffe aus der Säugetierpaläontologie für das Pliozän und Pleistozän Deutschlands
}

\author{
Wighart v. Koenigswald \& Wolf-Dieter Heinrich ${ }^{*}$
}

Einleitung: Die biostratigraphische Gliederung des Quartärs in Mitteleuropa erfolgte nach wechselnden Ansätzen, und entsprechend groß ist die Zahl der benutzen Termini. Zunächst wurde versucht, Stufen in der Entwicklung der Säugetierfaunen nach typischen Fundorten zu benennen und diese in einer zeitlichen Reihenfolge zu ordnen (z.B. KRETZOI 1962, 1969, JánOSSY 1969). Eine Korrelation mit den Glazialen und Interglazialen wurde zwar immer wieder versucht, brachte aber große Schwierigkeiten, weil die Faunen weitgehend aus den unvergletscherten Gebieten stammten und deswegen keine Korrelationen durch klare Superpositionen gegeben waren. Schon in der Faunenabfolge zeigte sich, dass die Zahl der Interglaziale, wie sie die klassische Gliederung anbot, nicht ausreichte, um die verschiedenen warmzeitlichen Faunen einzuordnen. Die Bohrungen in der Tiefsee zeigten dann sehr bald, dass die Zahl der Oszillationen weit höher sind, als von PENCK \& BRÜCKNER (1909) angenommen.

Die biostratigraphische Gliederung des Jungtertiärs konnte dadurch verfeinert werden, dass die Grenzen der einzelnen Stufen durch das erste bzw. letzte Auftreten (FAD = First Appearance Date bzw. LAD = Last Appearance Date) charakteristischer Säugetierarten definiert wurden. Dabei wurden Zeitspannen von rund 1 Million Jahren durch evolutive Veränderungen im Artbestand sowie durch gelegentliche Einwanderungswellen unterschieden (LinDSAY et al. 1989). Diese verfeinerte Methode wurde auch für die Gliederung des Quartärs angewendet (z.B. Guerin 1982, 1990, HoraceK \& LozeK 1988, FeJfar \& HeINRICh 1990), aber im Quartär treten zwei Probleme auf.

Zum einen ist die Gesamtdauer von nur rund 2 Millionen Jahren zu kurz, um evolutive Veränderungen zu erwarten, die eine Gliederung in der erwünschten Feinheit erlauben. In der Tat gibt es nur wenige Gattungen, in denen fassbare evolutive Veränderungen $\mathrm{zu}$ beobachten sind. Um diese Schwierigkeit zu umgehen, wird auch das gemeinsame Vorkommen von verschiedenen Arten, also deren zeitliches Überlappen zur Charakterisierung biostratigraphischer Zonen verwendet.

Die zweite Schwierigkeit wird durch die starken klimatischen Veränderungen verursacht, die zumindest in Mitteleuropa jeweils mit einem fast vollständigen Austausch der größeren Pflanzenfresser verbunden sind. Da die klimatischen Oszillationen mit einer Periodizität von rund 100.000 Jahren erfolgte, ist zu erwarten, dass zahlreiche Arten weitaus länger als nur in einem der zu charakterisierenden Zeitabschnitte vorkommen (KoENigswald \& HeINRICH 1999). Aus diesem Grund lassen sich kaltzeitliche bzw. warmzeitliche Faunen unterschiedlicher Zyklen nicht immer voneinander unterscheiden. In der Fauna ist das offensichtlich und gilt eventuell auch für die Zusammensetzung der Vegetation.

Der vielfache Faunenaustausch betrifft besonders die großen Pflanzenfresser, und deswegen ist das Vorkommen der auffälligen Formen, wie der Elefanten, Nashörner etc. zeitlich diskontinuierlich und zur Gliederung nur begrenzt geeignet. Bei den Kleinsäugern sind es die euryöken Formen, wie die Wühlmäuse, die zur Gliederung herangezogen werden können. Da nur relativ wenig Arten neu auftreten, muss auch das Verschwinden der Arten (LAD) zur Gliederung benutzt werden, obwohl darin

\footnotetext{
*Anschrift des Verfassers: Prof. Dr. Wighart v. Koenigswald, Institut für Paläontologie, Universität Bonn, Nussallee 8, 53115 Bonn, Email: Koenigswald@uni-bonn.de; Dr. Wolf-Dieter HeInRICH, Museum für Naturkunde, Humboldt-Universität Berlin, Invalidenstrasse, 10099 Berlin, Email: wolfdieter.heinrich@museum.hu-berlin.de
} 
immer eine gewisse Unsicherheit liegt und regionale Unterschiede bedeutend sein können. Man kann, besonders seit dem Mittelpleistozän, eine zunehmende Verarmung in der Säugetierfauna feststellen. Ganz allgemein zeichnet sich in den Faunen der ökologische Wandel weit stärker ab als der zeitliche Unterschied. Bei dem ökologischen Wandel spielen zwar die Änderungen in der Temperatur eine große Rolle, aber für Mitteleuropa sind die Verschiebungen im Feuchtigkeitsregime von mindestens ebenso großer Bedeutung. Während der Interglaziale nimmt der ozeanische Einfluss stark zu, weil der Meeresspiegel hoch stand. Dagegen verstärkt sich der kontinentale Einfluss während der Kaltzeiten (Koenigswald 1999, 2002). Der jeweilige Einfluss ist natürlich in Frankreich bzw. in Ungarn stärker oder schwächer. Dieser regionale Unterschied ist gerade in Europa sehr groß. Mitteleuropa war stets nur ein temporäres Verbreitungsgebiet der warmzeitlichen bzw. kaltzeitlichen Faunen. Die Kerngebiete, aus denen die Faunen jeweils neu eingewandert sind, lagen im Mittelmeergebiet bzw. im östlichen Europa und in Sibirien. Das hat zur Folge, dass das erste Auftreten mancher Gattungen etwa von Elephas oder Arvicola im Mittelmeergebiet möglicherweise wesentlich früher erfolgte als in Mitteleuropa. Diese regionalen Unterschiede sind sehr gravierend und erschweren eine biostratigraphische Korrelation über größere geographische Distanzen.

Trotz dieser besonderen Schwierigkeiten im Quartär wurde eine Gliederung erarbeitet, deren Präzision aber sehr unterschiedlich ist. Besonders im Altpleistozän reicht die Dichte der Fundstellen nicht aus, um eine gewünschte Zuverlässigkeit in der biostratigraphischen Gliederung zu geben.

Die Untergrenze des Pleistozäns wird in der Säugetierstratigraphie mit der Oberkante des Olduvay Chron bei etwa 1,8 Ma gleichgesetzt. Da z.B. in der Paläobotanik auch Teile des Pliozäns oberhalb der Gauss/Matuyama Grenze zum Pleistozän gerechnet werden, werden auch hier die entsprechenden biostratigraphischen Begriffe aus der Säugetierstratigraphie des Neogens (MN 14 - MN 17) mit aufgelistet. Das Mittelpleistozän beginnt nach allgemeiner Übereinkunft an der Brunhes/Matuyama Grenze und das Jungpleistozän mit dem letzten Interglazial, dem Eem.

Eine Übersicht über die wichtigsten stratigraphischen Begriffe für Mitteleuropa und ihre grobe zeitliche Korrelation wird in Tabelle 1 gegeben.

\title{
Biostratigraphical Terms from Mammal Palaeontology for the Pliocene and Pleistocene in Germany
}

\author{
Keywords: Bio-Stratotypes, Quaternary, Mammals, Germany
}

The biostratigraphical subdivision of the Quaternary in central Europe has followed various approaches, and its terminology is correspondingly large. At first, stages in the development of mammal fauna were named after the sites of typical finds and arranged chronologically (e.g. KRETZOI 1962, 1969, JÁNOSSY 1969). Repeated efforts to correlate them with glacial and interglacials were difficult, however, because most fauna came from unglaciated regions, and no correlations based on well-defined superpositions were possible. The faunal succession had already shown that the number of interglacials in the classic system was not enough to explain the different warm-stage faunas. Deep-sea boreholes soon showed that the number of oscillations was much higher than PENCK \& BRÜCKNER (1909) had assumed. The biostratigraphical subdivision of the Late Tertiary was refined by defining the boundaries of the individual stages by first and last appearances of characteristic species of mammals (FAD = First Appearance Date, LAD $=$ Last Appearance Date). Time ranges of about one million years are distinguished by evolutive changes in the species population and by occasional immigration waves (LINDSAY et al. 1989). This more refined method of subdivision was also applied to the Quaternary (e.g. GuERIN 1982, 1990, HoraceK \& LOzEK 1988, FeJFAR \& HeINRICH 1990), but two problems occur in the Quaternary. First, a duration of only about two million years is too short to expect evolutive changes that allow the desired detailed subdivision. Indeed there are only few genera that display tangible evolutive changes. To bypass this difficulty, the con- 
currence of different species, i.e. their overlap in time, is used to characterise biostratigraphical zones. The second difficulty is due to major climatic changes, each of which was associated - in central Europe at least - with the complete replacement of the larger herbivores. Because the climate oscillations occurred with a periodicity of about 100,000 years, it is likely that many species are found in more than just one of the time ranges (KOENIGSWALD \& HEINRICH 1999). For this reason, it is not always possible to distinguish cold-stage or warm-stage faunas of subsequent cycles. This difficulty is obvious in the case of fauna and may also apply to the vegetation composition.

Multiple faunal replacement affected the large herbivores in particular: elephants and rhinoceroses, for example, do not occur continuously and are of limited value for biostratigraphical purposes. Of the small mammals, euryoecious species such as voles may be appropriate for biostratigraphy. Since only relatively few new species occur, last appearance dates have to be applied as well, although this involves some uncertainty and regional variations may be significant. An increasing impoverishment of mammal fauna is evident, especially since the Middle Pleistocene.

With regard to fauna, it is generally easier to recognise ecological change than change through time. Although temperature variations are a major factor in ecological change, shifts in the humidity regime are at least equally important in central Europe. During the interglacials, marine influence increased strongly because sea-level was high. By contrast, continental influence increased during the cold stages (KoENIGSWALD 1999, 2002). Both influences are, of course, stronger or weaker in France or in Hungary. This regional difference is very great, especially in Europe. Central Europe has always been only a temporary area of distribution for warm-stage or cold-stage fauna. The source areas of faunal migrations were in the Mediterranean, eastern Europe, or Siberia. As a result, the first occurrence of some genera - Elephas or Arvicola, for example - may have been much earlier in the Mediterranean than in central Europe. Such regional differences are significant and impede biostratigraphical correlation over great distances.

In spite of these specific differences in the Quaternary a subdivision was attempted, but its precision varies greatly. For the Lower Pleistocene in particular, there are not enough localities to achieve the desired biostratigraphical reliability.

In mammal palaeontology, the basal boundary of the Pleistocene is positioned at about $1.8 \mathrm{Ma}$, at the top of the Olduvai chron. In palaeobotany, parts of the Pliocene above the Gauss/Matuyama boundary are assigned to the Pleistocene, which is why the corresponding biostratigraphical terms from Neogene mammal stratigraphy (MN $14-\mathrm{MN} 17)$ are also listed here. It is generally agreed that the Middle Pleistocene begins at the Brunhes/Matuyama boundary, and the Upper Pleistocene at the last interglacial, the Eemian stage. Table 1 gives an overview of the most important stratigraphical terms for central Europe and their approximate time correlation.

\section{Biostratigraphische Begriffe für das Pliozän}

\section{Ruscinium / MN 14 und MN 15}

\section{Erstbeschreibung und Definition}

Das Ruscinium ist eine Superzone des Pliozäns. Der Begriff wurde von KRETzOI (1962) eingeführt und durch die Faunen von Serart d'en Vaquer und Perpignan im Roussillon definiert.

Das Ruscinium wird durch verarmte Hipparion-Faunen (Liegendes) und durch die Csarnóta-Faunen (Hangendes) biostratigraphisch begrenzt (Kretzoi 1962). Nach Berggreen \& VAn Couvering (1974) liegt das Ruscinium biochronologisch zwischen dem Turolium und dem Villafranchium. Es umfasst die MN Zonen 14 und 15 (STEININGER et al. 1990).

FAD: Trilophomys und LAD: Ruscinomys

\section{Typuslokalität}

Typuslokalität des Rusciniums ist Serrat d'en Vacquer, Süd-Frankreich (KRETzOI 1962). (Georef. $\left.02^{\circ} 52^{`} \mathrm{E}-42^{\circ} 40^{`} \mathrm{~N}\right)$. Mein \& Aymar (1984) und Mein (1990) geben eine Faunenliste. 
Tab. 1. Korrelation der Begriffe auf den verschiedenen stratigraphischen Ebenen.

Tab. 1: Correlation of biostratigraphical terms on different stratigraphical levels.

\begin{tabular}{|c|c|c|c|c|c|c|}
\hline Geologische & Superzonen $^{1)}$ & Biozonen & \multicolumn{4}{|c|}{ Andere Begriffe ${ }^{2) 344)}$} \\
\hline Holozän & \multirow{4}{*}{$\begin{array}{l}\text { Toringium } \\
\text { (Arvicola- } \\
\text { Microtus- } \\
\text { Superzone) }\end{array}$} & $\begin{array}{l}\text { Arvicola terrestris- } \\
\text { Faunen }\end{array}$ & \multirow[b]{3}{*}{ Steinheimium } & & $\begin{array}{l}\mathrm{Q} \\
4\end{array}$ & $\begin{array}{c}\mathrm{MQ} \\
2\end{array}$ \\
\hline $\begin{array}{c}\text { Jung- } \\
\text { Pleistozän }\end{array}$ & & & & $\begin{array}{c}\text { MNQ } \\
26 \\
\text { MNQ } \\
25\end{array}$ & $\begin{array}{l}\mathrm{Q} \\
3\end{array}$ & \\
\hline \multirow{3}{*}{$\begin{array}{l}\text { Mittel- } \\
\text { Pleistozän }\end{array}$} & & $\begin{array}{c}\text { Jüngere } \\
\text { Arvicola cantianus- } \\
\text { Faunen }\end{array}$ & & $\begin{array}{l}\text { MNQ } \\
24-23\end{array}$ & & \\
\hline & & $\begin{array}{c}\text { Ältere } \\
\text { Arvicola cantianus- } \\
\text { Faunen } \\
\end{array}$ & Mosbachium & $\begin{array}{c}\text { MNQ } \\
22\end{array}$ & & \\
\hline & \multirow[t]{2}{*}{$\begin{array}{l}\text { Biharium } \\
\text { (Microtus - } \\
\text { Mimomys } \\
\text { Superzone) }\end{array}$} & $\begin{array}{c}\text { Mimomys savini-Faunen } \\
\text { (=Jungbiharium) }\end{array}$ & $\begin{array}{l}\text { Cromerium } \\
\text { s. str. }\end{array}$ & $\begin{array}{l}\text { MNQ } \\
21-19\end{array}$ & $\begin{array}{l}\mathrm{Q} \\
2\end{array}$ & $\begin{array}{c}\mathrm{MQ} \\
1\end{array}$ \\
\hline $\begin{array}{c}\text { Alt- } \\
\text { Pleistozän } \\
(1,8 \mathrm{Ma})\end{array}$ & & $\begin{array}{l}\text { Mimomys pusillus - } \\
\text { Mimomys savini-Zone } \\
\text { (Altbiharium) }\end{array}$ & p.p. Betfia & & $\begin{array}{l}\mathrm{Q} \\
1\end{array}$ & \\
\hline \multirow{7}{*}{ Pliozän } & \multirow{3}{*}{$\begin{array}{l}\text { Villanyium } \\
\text { (Borsodia - } \\
\text { Villanyia- } \\
\text { Superzone) }\end{array}$} & $\begin{array}{c}\text { Mimomys pliocaenicus- } \\
\text { Zone }\end{array}$ & & MN17 & & \\
\hline & & $\begin{array}{c}\text { Mimomys polonicus- } \\
\text { Zone }\end{array}$ & & MN16 & & \\
\hline & & $\begin{array}{l}\text { Mimomys hassiacus - } \\
\text { Mimomys stehlini-Zone }\end{array}$ & & & & \\
\hline & \multirow{4}{*}{$\begin{array}{l}\text { Ruscinium } \\
\text { (Trilophomys- } \\
\text { Ruscinomys- } \\
\text { Superzone) }\end{array}$} & $\begin{array}{c}\text { Mimomys hassiacus - } \\
\text { Mimomys gracilis-Zone } \\
\text { Mimomys occitanus- } \\
\text { Zone }\end{array}$ & & \multirow[t]{2}{*}{ MN15 } & & \\
\hline & & Mimomys davakosi-Zone & & & & \\
\hline & & $\begin{array}{l}\text { Promimomys cor-Zone } \\
\text { Prom. moldavicus- Zone }\end{array}$ & & \multirow[t]{2}{*}{ MN14 } & & \\
\hline & & $\begin{array}{c}\text { Promimomys insuliferus- } \\
\text { Zone }\end{array}$ & & & & \\
\hline
\end{tabular}

1) Superzonen und Biozonen nach FEJFAR et al. (1998)

2) MN 14-17 nach MeIN in Lindsay et al. (1989) und MNQ19-26 nach GuERIN $(1982,1990)$

3) Q1-Q4 nach HoRAcEK \& LOZEK (1988)

4) MQ1 und MQ2 nach FEJFAR \& HEINRICH (1990) 


\section{Biostratigraphische Zuordnung}

In der ursprünglichen Fassung ist das Ruscinium (Kretzor 1962, s. auch Kretzor 1985) biochronologisch nicht eindeutig definiert. Eine bessere Kennzeichnung ermöglichen die stratigraphischen Reichweiten von Nagetieren. Das Ruscinium lässt sich durch das gemeinsame Auftreten von Trilophomys und Ruscinomys biochronologisch eindeutig als Trilophomys-Ruscinomys-Superzone charakterisieren (Fejfar \& HeinRICh 1990, Fejfar et al. 1997, 1998) und dadurch vom vorangehenden Turolium ( $\mathrm{Va}$ lerymys-Hispanomys-Superzone) und nachfolgenden Villanyium (Borsodia-Villanyia-Superzone) gut abgrenzen. Die Trilophomys-Ruscinomys-Superzone korrespondiert mit den MN Zonen 14 und 15. Die graduelle Evolution von Promimomys und Mimomys liefert Zeitmarken, die die Grundlage für die Zonengliederung des Rusciniums (Trilophomys-Ruscinomys-Superzone) sind. Am Ende des Rusciniums verschwinden die letzten Vertreter der Gattung Mimomys, die keinen Zahnzement in den Falten haben. Die Untergrenze der Trilophomys-Ruscinomys-Superzone ist durch das FAD von Trilophomys, die Obergrenze durch das Verschwinden von Ruscinomys sowie durch das FAD von Borsodia gekennzeichnet, das zugleich den Beginn des Villanyiums markiert.

Das Ruscinium entspricht dem Unterpliozän (SteInINGER et al. 1990).

\section{Untergliederung}

Das Ruscinium wird in die Promimomys insuliferus- (MN 14 a) und Promimomys cor-Stufe (MN 14b) sowie in die Miomys davakosi- (MN 15a) und Mimomys hassiacus-Mimomys gracilis (MN 15b) unterteilt (FEJFAR et al. 1998)

\section{Zugehörige Faunen aus Mitteleuropa und angrenzenden Gebieten}

Gundersheim 3, 4 (RP); Wölfersheim (HE); ferner Montpellier (F), Villeneuve (F), Sète F), Celadas 9 (E), Podlesice (PL), Weze (PL) und Ptolemais 1 (GR). Von Fejfar (2001) werden Gundersheim 3 und 4 (RP) sowie Wölfersheim (HE) in das untere Villanyium (MN 16a) gestellt, was jedoch nicht gesichert ist.

\section{Promimomys-'Stufe'/MN 14}

\section{Erstbeschreibung und Definitionen}

Die Promimomys-'Stufe' wurde von Fejfar \& Heinrich (1981b) aufgestellt und ist der ältere Teil des Rusciniums. Sie deckt die gesamte Zeit des Vorkommens von Promimomys, einem Nager, im zentralen und westlichen Europa ab (Fejfar et al. 1998: 535, Tab. 1). Die Promimomys-'Stufe' ist gleichbedeutend mit der MN 14.

Die Neogene Mammal Zones wurden von Mein (1975) eingeführt, um Säugetierfaunen aufgrund ihres ähnlichen Artbestandes zu charakterisieren. MeIN (1990) überarbeitet die Gliederung und gibt neben den typischen Arten auch an, welche Arten erstmals in der entsprechenden Zone auftreten beziehungsweise letztmalig vorkommen.

\section{Typuslokalität}

Von FeJfar \& Heinrich (1981b) wurde keine Typuslokalität festgelegt. Die Fauna von Podlesice in Südpolen (Georef. $19^{\circ} 34^{\circ} \mathrm{E}-51^{\circ} 34^{\circ} \mathrm{N}$ ) kann für diesen Zeitraum als typisch angesehen werden (MeIN 1990).

\section{Biostratigraphische Zuordnung}

Unteres Ruscinium, Unter-Pliozän

\section{Zugehörige Faunen aus Mitteleuropa und angrenzenden Gebieten}

Villeneuve (F), Vendargues (F), Celadas 9 (E), Terrats (E), Podlesice (PL), Kardia (GR), Ptolemais 1 (GR), Ostramos 1, 9, $13(\mathrm{H})$. 


\section{Promimomys-Ruscinomys-'Stufe' / MN 14}

\section{Erstbeschreibung und Definitionen}

Die Promimomys-Ruscinomys-'Stufe' wurde von FeJfar \& HeINRICH (1987) aufgestellt und umfasst den älteren Teil des Rusciniums. Sie ist gleichbedeutend mit MN 14, die von MeIN (1975) aufgestellt und MeIN (1990) revidiert wurde. Diese Faunenstufe ist durch das gemeinsame Auftreten der Nagetiere Promimomys und Ruscinomys gekennzeichnet. Für sie wurde auch der Begriff Montpellierium vorgeschlagen, eine Bezeichnung, die von GAUDRY (1878) eingeführt wurde (Einzelheiten bei FEJFAR \& HEINRICH 1987).

\section{Typuslokalität}

Celadas 9 (E) (Fejfar \& HeInRICh 1987). Als typische Fauna führt Mein (1990) die Fauna aus Podlesice in Südpolen (Georef. 19³4'E - 51 $34^{\circ} \mathrm{N}$ ) an.

\section{Biostratigraphische Zuordnung}

Unteres Ruscinium, Unter-Pliozän.

\section{Promimomys insuliferus-Zone/MN 14a}

\section{Erstbeschreibung und Definitionen}

Der Zonenbegriff wurde von FEJFAR \& HeINRICH (1987) eingeführt. Er kennzeichnet den Zeitabschnitt des Rusciniums, der durch die stratigraphische Reichweite des Nagetieres Promimomys insuliferus gekennzeichnet ist.

\section{Typuslokalität}

Als Typuslokalität wurde Podlesice (PL) (Agadzhanyan \& Kowalski 1978) festgelegt (Fejfar \& HEINRICH 1987, 1990).

\section{Biostratigraphische Zuordnung}

Unteres Unter-Ruscinium, Unter-Pliozän.

\section{Zugehörige Faunen aus Mitteleuropa und angrenzenden Gebieten}

Hautimagne (F), Villeneuve (F), Montpellier (F), Celadas 9 (E) und Terrats (E).

\section{Promimomys moldavicus-Zone/MN 14b}

\section{Erstbeschreibung und Definitionen}

Die Promimomys moldavicus-Zone wurde von FeJfar \& HeInrich (1987) aufgestellt. Sie charakterisiert den Zeitabschnitt des Rusciniums, der durch die stratigraphische Reichweite von Promimomys moldavicus gekennzeichnet ist.

\section{Typuslokalität}

Als Typuslokalität wurde Malusteni (RO) (Simionescu 1930, Kormos 1932) bestimmt (FeJfar \& HEINRICH 1987, 1990).

\section{Biostratigraphische Zuordnung}

Oberes Unter-Ruscinium, Unter-Pliozän. 


\section{Bemerkungen}

Bei den Funden von Malusteni (RO) handelt es sich um stark abgekaute Backenzähne, die zunächst zu Mimomys (Kormos 1932), dann zu Promimomys (Kretzor 1956, FeJfar et al. 1998) gestellt wurden (s. dazu auch Radulesco \& Samson 1989). Nach FeJfar et al. (1998) liegt dort Promimomys cor vor. Daher ist die Bezeichnung Promimomys moldavicus-Zone durch den Begriff Promimomys cor-Zone zu ersetzen.

\section{Promimomys cor-Zone/MN 14b}

\section{Erstbeschreibung und Definitionen}

Die Promimomys cor-Zone wurde von FEJFAR et al. (1998) eingeführt. Sie charakterisiert den Zeitabschnitt des Rusciniums, der durch die stratigraphische Reichweite von Promimomys cor gekennzeichnet ist, die von Csarnóta 2 (H) (KRETzor 1959, 1962) beschrieben wurde.

\section{Typuslokalität}

Noch nicht festgelegt.

\section{Biostratigraphische Zuordnung}

Oberes Unter-Ruscinium, Unter-Pliozän.

\section{Zugehörige Faunen aus Mitteleuropa und angrenzenden Gebieten}

siehe Promimomys moldavicus-Zone.

\section{Mimomys-Ruscinomys-Zone/MN 15}

\section{Erstbeschreibung und Definitionen}

Die 'Stufe' wurde von FeJfar \& HeinRich (1987) eingeführt und durch das gemeinsame Vorkommen der Nagetiergattungen Mimomys und Ruscinomys definiert.

\section{Typuslokalität}

Als Typuslokalität wurde Serrat d'en Vaquer (F) (Mein \& Aymar 1984) festgelegt (FeJFar \& HeinRich 1987).

\section{Biostratigraphische Zuordnung}

Oberes Ruscinium, unteres Ober-Pliozän.

\section{Zugehörige Faunen aus Mitteleuropa und angrenzenden Gebieten}

Gundersheim 3, 4 (RP); Wölfersheim (HE); ferner: Serrat d'en Vacquer (F), Sète (F), Orrios 3 (E), Layna (E), Ptolemais 3 (GR).

\section{Mimomys davakosi-Zone/MN 15a}

\section{Erstbeschreibung und Definitionen}

Die Zone wurde von FeJfar \& HeInRICh (1987) aufgestellt. Sie ist durch die stratigraphische Reichweite von Mimomys davakosi charakterisiert.

\section{Typuslokalität}

Als Typuslokalität wurde Ptolemais 3 (GR) (VAn DE WeERd 1979) festgelegt (FeJfar \& HeInRICh 1987, 1990). 


\section{Biostratigraphische Zuordnung}

Unteres Ober-Ruscinium, unteres Ober-Pliozän.

4 Zugehörige Faunen aus Mitteleuropa und angrenzenden Gebieten

Orrios 1 (E), El Arquillo 3 (E), La Gloria 3 (E), Serrat d'en Vaquer (F).

\section{Mimomys occitanus-Zone/MN 15b}

\section{Erstbeschreibung und Definitionen}

Die Zone ist von FEJFAR \& HEINRICH (1987) eingeführt und durch die stratigraphische Reichweite von Mimomys occitanus definiert worden.

\section{Typuslokalität}

Als Typuslokalität wurde Sète (F) (Thaler 1966) festgelegt (FeJfar \& HeInRICH 1987, 1990).

\section{Biostratigraphische Zuordnung}

Oberes Ober-Ruscinium, unteres Ober-Pliozän.

\section{Zugehörige Faunen aus Mitteleuropa und angrenzenden Gebieten}

Gundersheim 3, 4 (RP), Wölfersheim (HE); ferner La Gloria 2 (E), Layna (E), Nimes (F), Csarnóta 2 (H), Ivanovce A, B (SK), Weze (PL).

\section{Bemerkungen}

Neuuntersuchungen von Mimomys occitanus von Sète (F) haben gezeigt, dass diese Art nicht zu Mimomys sondern zur Gattung Dolomys gehört (Maul 1996). Daher wurde die Mimomys occitanusZone aufgegeben und durch die Mimomys hassiacus-Mimomys gracilis-Zone ersetzt (Einzelheiten s. FEJfAR et al. 1998). Von FeJfar (2001) werden Gundersheim 3 und 4 (RP) sowie Wölfersheim (HE) in das untere Villanyium (MN 16a) gestellt, was jedoch nicht gesichert ist.

\section{Mimomys hassiacus-Mimomys gracilis-Zone/MN 15b}

\section{Erstbeschreibung und Definitionen}

Die Zone ist von FeJfar et al. (1998) eingeführt worden. Sie kennzeichnet den Zeitraum des späten Rusciniums, der durch das gemeinsame Vorkommen von Mimomys hassiacus und Mimomys gracilis gekennzeichnet ist.

\section{Typuslokalität}

Noch nicht festgelegt.

\section{Biostratigraphische Zuordnung}

Oberes Ober-Ruscinium, unteres Ober-Pliozän.

\section{Zugehörige Faunen aus Mitteleuropa und angrenzenden Gebieten}

wie Mimomys occitanus-Zone 


\section{Villanyium/MN 16 und MN 17}

\section{Erstbeschreibung und Definitionen}

Das Villanyium ist eine Superzone und umfasst den jüngeren Teil des Pliozäns. Der Begriff geht auf Kretzor (1941) zurück, der das Cromerium damaliger Fassung in die Unterstufen Villanyium, Biharium und Mosbachium untergliederte. Dem Villanyium wurden die Faunen von Villány $(\mathrm{H})$, Csarnóta $(\mathrm{H})$ und Beremend $(\mathrm{H})$ zugewiesen. In dieser ursprünglichen Fassung ist das Villanyium biostratigraphisch heterogen, da ruscinische (z.B. Csárnota $2(\mathrm{H})$ und villanyische Faunen (z.B. Villány $3(\mathrm{H})$, Beremend 1,3 und $5(\mathrm{H})$ ) zusammengefasst wurden (FEJfar \& HoraceK 1983).

Die biochronologische Uneinheitlichkeit des Villanyiums legte Emendationen nahe (Einzelheiten dazu bei Fejfar \& Heinrich 1981a, b, 1983, 1987, 1990, Fejfar \& HoraceK 1983). Die Rodentier-Biochronologie definiert das Villanyium heute als Zeitabschnitt, in dem die Nagetiergattungen Borsodia und Villanyia gemeinsam auftreten (Borsodia-Villanyia-Superzone: FeJfar \& HeInRICH 1990, FeJfar et al. 1997, 1998). Der Beginn des Villanyiums ist durch das FAD von Borsodia, das Ende durch das FAD von Microtus (Allophaiomys) festgelegt, mit dem das Biharium (Microtus-Mimomys-Superzone) beginnt. Außerdem stirbt Villanyia am Ende dieses Zeitabschnittes aus. Am Anfang des Villanyiums erscheinen erstmals zahlreiche Mimomys-Arten, deren Backenzähne Zahnzement aufweisen. Am Ende des Villanyiums stirbt Villanyia aus, was eine weitere, wenn auch weniger verlässliche Zeitmarke bietet. Das als Borsodia-Villanyia-Superzone definierte Villanyium fällt zeitlich mit den MN Zonen 16 und 17 zusammen, was gut mit der Neogene mammal faunal biozonation Europas (LiNDSAY et al. 1989, Fahlbusch \& Mein 1989, Steininger et al. 1990) übereinstimmt.

Bei den Säugetierpaläontologen herrscht weitgehende Übereinstimmung darüber, dass sich das Villanyium, das mit Rodentiern definiert ist, und das Villafranchium, das mit Großsäugetieren gekennzeichnet wurde, zeitlich weitgehend überschneiden. Daher wurde von FEJFAR et al. (1998) vorgeschlagen, diese Zeitspanne vorläufig als Villanyium/Villafranchium (Villanyian/Villafranchian land mammal age) zu bezeichnen, bis bessere Kriterien für die Kennzeichnung der beiden stratigraphischen Einheiten zur Verfügung stehen (Einzelheiten s. FEJFAR et al. 1998: 536).

FAD: Borsodia und LAD: Villanyia

\section{Typuslokalität}

Karstspaltenfüllung Villány 3 im Villányer Gebirge, Süd-Ungarn (KRETZOI 1941). (Georef. 1857` E $\left.-45^{\circ} 53^{\circ} \mathrm{N}\right)$.

\section{Biostratigraphische Zuordnung}

Ober-Pliozän

\section{Vergleichbare Faunen aus Mitteleuropa und angrenzenden Gebieten}

Uhlenberg/Dinkelscherben (BY), Solnhofen (BY), Deinsdorf (BY), Schernfeld (BY), Schambach (BY), Erpfingen 2 (BW), ferner Seynes (F), Balaruc 2 (F), Les Etouaires (F), Arondelli-Triversa (I), Hajnácka (SK), Vcelare (SK), Beremend 1, 2 (H), Ostramos $3(\mathrm{H})$.

\section{Unterteilung}

Borsodia-Dolomys Zone (MN 16) und Lagurodon-Villanyia Zone (MN 17). Das Villanyium wird in die Mimomys hassiacus-Mimomys stehlini-Zone, Mimomys polonicus-Zone und die Mimomys pliocaenicus-Zone (FEJFAR et al. 1998).

\section{Borsodia-Dolomys-'Stufe'/MN 16}

\section{Erstbeschreibung und Definitionen}

Die Borsodia-Dolomys-'Stufe' wurde von FeJfar \& Heinrich (1981a, b) aufgestellt. Sie bildet den 
älteren Abschnitt des Villanyium/Villafranchiums und entspricht dem MN 16, das von MeIN (1975) eingeführt und MeIn (1990) revidiert wurde. Diese Faunenstufe ist durch das erste Auftreten von Leptobos, Mammuthus und Equus sowie durch die Wühlmäuse Borsodia und Dolomys gekennzeichnet, die hochkronige Molaren besitzen.

\section{Typuslokalität}

Als Typuslokalität wurde Hajnácka (SK) (Fejfar 1964) vorgeschlagen (FEJFAR \& HeINRICH 1981b). Eine weitere typische Fauna liegt aus Arondelli-Triversa (Villafranca d'Asti) in Norditalien (Georef. $08^{\circ} 00^{`} \mathrm{E}-44^{\circ} 55^{\circ} \mathrm{N}$ ) vor (MeIN 1989, FeJfar 2001). Für das Villafranchium ist Villafranca d'Asti (I) die Typuslokalität (PARETo 1865).

\section{Biostratigraphische Zuordnung}

Unteres Villanyium, Ober-Pliozän.

\section{Zugehörige Faunen aus Mitteleuropa und angrenzenden Gebieten}

Reuverton in der Niederrheinischen Bucht, Hambach (NRW), ferner Seynes (F), Vialette (F), Les Etouaires (F), Chagny 2 (F), Balaruc 2 (F), Arondelli-Triversa (I), San Giusto (I), Ostramos 7 (H), Rembielice Królewskie (PL) und Deutsch-Altenburg 21(A).

\section{Mimomys hajnackensis-Zone/MN 16a}

\section{Erstbeschreibung und Definitionen}

Die Zone ist von FEJFAR \& HEINRICH (1981a) eingeführt und durch die stratigraphische Reichweite von Mimomys hajnackensis definiert worden.

\section{Typuslokalität}

Als Typuslokalität wurde Hajnácka in der Slowakei (FeJfar 1964) festgelegt (FeJfar \& HeInRICH 1990).

\section{Biostratigraphische Zuordnung}

Unteres Unter-Villanyium, unteres Ober-Pliozän.

\section{Zugehörige Faunen aus Mitteleuropa und angrenzenden Gebieten}

Orrios 2, 3 (E), Seynes (F), Balaruc 2 (F), Vialette (F), Arondelli-Triversa (I), Arcille (I).

\section{Bemerkungen}

Nach neueren Untersuchungen ist Mimomys hajnackensis ein jüngeres Synonym von Mimomys hassiacus (FEJFAR et al. 1998). Daher wurde die Mimomys hajnackensis-Zone aufgegeben und durch die Mimomys hassiacus-Mimomys stehlini-Zone ersetzt (Einzelheiten s. FeJfar et al. 1998).

\section{Mimomys hassiacus-Mimomys stehlini-Zone/MN 16a}

\section{Erstbeschreibung und Definitionen}

Die Zone ist von FEJFAR et al. (1998) eingeführt und durch das gemeinsame Vorkommen von Mimomys hassiacus und Mimomys stehlini definiert worden.

\section{Typuslokalität}

Noch nicht festgelegt. 


\section{Biostratigraphische Zuordnung}

Unteres Unter-Villanyium, unteres Ober-Pliozän.

\section{Zugehörige Faunen aus Mitteleuropa und angrenzenden Gebieten}

siehe Mimomys hajnackensis-Zone

\section{Bemerkungen}

Die Mimomys hassiacus-Mimomys stehlini-Zone ersetzt die Mimomys hajnackensis-Zone, da Mimomys hajnackensis ein jüngeres Synonym von Mimomys hassiacus ist (Einzelheiten s. Fejfar et al. 1998).

\section{Mimomys polonicus-Zone/MN 16b}

\section{Erstbeschreibung und Definitionen}

Die Zone ist von FeJfar \& HeinRich (1981a) aufgestellt worden. Sie ist durch die stratigraphische Reichweite von Mimomys polonicus definiert.

\section{Typuslokalität}

Als Typuslokalität wurde Rembielice Krolewskie (PL) (Kowalski 1960) festgelegt (FeJfar \& HeinRICH 1990).

\section{Biostratigraphische Zuordnung}

Oberes Unter-Villanyium, oberes Ober-Pliozän.

\section{Zugehörige Faunen aus Mitteleuropa und angrenzenden Gebieten} Concude (F), Village (F), Les Etouaires (F), Chagny 2 (F), Ostramos 7 (H), Montopoli (I).

\section{Lagurodon-Villanyia-'Stufe'/MN 17}

\section{Erstbeschreibung und Definitionen}

Die Lagurodon-Villanyia-'Stufe' wurde von FeJFAR \& Heinrich (1981a) aufgestellt, bildet den oberen Teil des Villanyiums und entspricht dem MN 17, das von MEIN (1975) aufgestellt und von MeIN (1990) revidiert wurde. Diese Faunenstufe ist durch die Wühlmäuse Lagurodon und Villanyia gekennzeichnet.

\section{Typuslokalität}

Als typische Fauna führt MeIN (1989) die Fauna aus Saint Vallier im Rhonetal (Georef. 0450`E $\left.45^{\circ} 11^{\circ} \mathrm{N}\right)$ an.

\section{Biostratigraphische Zuordnung}

Oberes Villanyium, oberes Ober-Pliozän.

\section{Zugehörige Faunen aus Mitteleuropa und angrenzenden Gebieten}

Schernfeld auf der Schwäbisch-Fränkischen Alb (BY), Uhlenberg/Dinkelscherben (BY), Solnhofen (BY), Schambach (BY), Deinsdorf (BY), Erpfingen 2 (BW), Moggaster Höhle in der Fränkischen Schweiz (BY), Rocca Neyra (F), Chilhac (F), Seneze (F), Tegelen (NL), Vcelare (SK), Kolinany 1, 2 (SK) Ostramos 3 (H), Olivola (I). 


\section{Mimomys pliocaenicus-Zone MN 17}

\section{Erstbeschreibung und Definitionen}

Die Zone ist zunächst von FeJFAR \& HeInRICH (1981a, 1987) ohne nähere Beschreibung ausgehalten und später durch die stratigraphische Reichweite von Mimomys pliocaenicus definiert worden (FEJFAR \& HEINRICH 1990).

\section{Typuslokalität}

Als Typuslokalität ist Villány 3 (H) (KRETzOI 1956) festgelegt worden (FeJFAR \& HeInRICH (1990).

\section{Biostratigraphische Zuordnung}

Oberes Villanyium, oberes Ober-Pliozän.

\section{Zugehörige Faunen aus Mitteleuropa und angrenzenden Gebieten}

Uhlenberg/Dinkelscherben (BY), Solnhofen (BY), Deinsdorf (BY), Schernfeld (BY), Schambach (BY); ferner Le Coupet (F), Seneze (F), Tegelen (NL), Plesivec 1 (SK), Kolinany 1, 2 (SK), Ostramos $3(\mathrm{H})$.

\section{Bemerkungen}

Die Mimomys pliocaenicus-Zone fällt zeitlich mit der MN Zone 17 (MeIN 1975, 1990) zusammen.

\section{Biostratigraphische Begriffe für das Quartär}

\section{Biharium}

\section{Erstbeschreibung und Definitionen}

Das Biharium bildet eine Superzone und umfasst zeitlich das ganze Altpleistozän sowie den älteren Teil des Mittelpleistozäns. Der Begriff wurde von KRETZOI (1941) geprägt und mit der Fauna von Betfia 2 (=Püspökfürdö, Episcopia) gekennzeichnet, in der Mimomys (Allophaiomys) erstmals auftaucht. Die ursprüngliche Fassung des Bihariums war sehr weit und schloss sowohl die Mimomys- wie die Arvicola-Faunen und damit sehr unterschiedliche faunengeschichtliche Etappen vom Pliozän bis Mittel-Pleistozän ein, ohne dass eine Obergrenze definiert wurde. Deswegen wurde eine Emendation vorgenommen (Fejfar 1976, Fejfar \& Heinrich 1981b, 1990). Sie haben das Biharium durch das gemeinsame Vorkommen der Wühlmausgattungen Mimomys und Microtus definiert. Die Basis des Bihariums ist durch das FAD von Microtus (Allophaiomys) gekennzeichnet, was der Basis des Pleistozäns entspricht. Die Obergrenze ist durch das Erscheinen von Arvicola festgelegt. In dieser Fassung kommt dem Biharium der Rang einer Superzone zu: Microtus-Mimomys -Superzone.

FAD: Microtus (Allophaiomys) und LAD: Mimomys savini

\section{Typuslokalität}

Bei der Beschreibung der Fauna von Betfia 2 (RO) (Georef. etwa $22 \mathrm{E}^{\circ}$ - $47 \mathrm{~N}^{\circ}$ ) bei der Stadt Biharia in Transylvanien (Bez. Bihor, ungarisch: Bihar) im heutigen Rumänien und der Einführung des Begriffs Biharium durch KRETzOI (1941) ist keine Typuslokalität festgelegt worden. Der Fauneninhalt der Spaltenfüllung Betfia 2 (RO) ist von KRETzoI (1941) und JÁNOSSY (1986) ausführlich beschrieben bzw. aufgelistet worden. KRETZOI (1965: 609) erwähnt, dass die biharischen Faunen im "StratotypenKomplex der Biharischen Stufe, dem Villányer Templomhegy (Kirchberg, Kalkberg),” in „N-S angeordneten Spalten” liegen. Daraufhin wurde die Lokalität Villány $8(\mathrm{H})$ mit den Schichten 9-12 und I - VII als Typuslokalität des Bihariums festgelegt (van DER Meulen 1973).

\section{Biostratigraphische Zuordnung}

Alt-Pleistozän und älteres Mittel-Pleistozän 


\section{Unterteilung und Synonyme}

Das Biharium (ursprüngliche Fassung) ist von Kretzor $(1956,1965)$ in eine Vielzahl von Zonen untergliedert worden, die in „Phasen” zusammengefasst wurden. Nach KrEtzor (1956: 653, Tab.7) bilden die Betfia-, Nagyharsanyhegy-, Templomhegy- und Tarkö-Phase das Altbiharium, die Vertesszöllös- und eine weitere nicht näher benannten Faunenphase das Jungbiharium. In einer Biozonation des Alt- und Mittelpleistozäns Mittel- und Westeuropas untergliederte van DER MEulen (1973: 109, Fig. 42) das Biharium in 4 Biozonen und 13 Subzonen, wobei von einer Unterteilung in ein Alt- und Jungbiharium Abstand genommen wurde. Eine weitere Untergliederung des Bihariums, die das FAD von Microtus (Allophaiomys) ignoriert und Faunen aus dem Villanyium einbezieht, hat RABEDER (1981) vorgelegt. Die Reichweite der späten Mimomys-Arten bietet eine gute Möglichkeit, das Biharium einfacher und wesentlich handhabbarer zu untergliedern (z. B. KoENIGSwald \& HeInRICH 1999). Nach Fejfar \& HeInrich (1990) ist das Biharium in Mitteleuropa in die Mimomys savini-Mimomys pusillus-Zone (Altbiharium) und die Mimomys savini-Zone (Jungbiharium) zu untergliedern (FEJFAR et al. 1998). Im Biharium unterscheidet GUERIN (1990) aufgrund der Nashörner die Zonen MNQ1921. FeJfar \& HeInRICh (1990) führten für das Biharium das Kürzel MQ1 zur Abgrenzung von den Faunen des Neogens (MN 1-17) ein. Für das Altbiharium verwenden HoraceK \& LozeK (1988) die Kurzbezeichnung Q1.

\section{Zugehörige Faunen aus Mitteleuropa und angrenzenden Gebieten}

Süssenborn (TH), Voigtstedt (ST), Mahlis (SN), Niederaußem (NRW), Neuleiningen 11 (RP), Sackdilling (BY), Weißenburg 7 (BY), Hohensülzen (RP), Prezletice (CZ), Koneprusy C 718 (CZ), Chlum 4-6 (CZ), Zirany (SK), Gombasek (SK), Mokrá 1 (CZ), Holstejn (CZ), West Runton (GB), Brielle 1 (NL), Maasvlakte NL), Bavel (NL), Les Valerots (F), Chagny 1 (F), Bourgarde 2 (F).

\section{Mimomys savini-Mimomys pusillus-Zone (Altbiharium)}

\section{Erstbeschreibung und Definitionen}

Mimomys savini-Mimomys pusillus-Zone, die von FeJfar \& HeINRICH (1990) aufgestellt wurde, ist durch das gemeinsame Vorkommen von Mimomys savini und Mimomys pusillus gekennzeichnet.

Das Biharium wurde als Biozone von Kretzor (1941) aufgestellt. Der Beginn des Altbihariums ist durch das erste Auftreten von Microtus (Allophaiomys) definiert. Zur Abgrenzung von jüngeren Faunen ist bedeutsam, dass Mimomys savini im Altbiharium noch zusammen mit anderen Mimomys -Arten auftritt (Mimomys pusillus, Mimomys reidi), wohingegen im Jungbiharium Mimomys savi$n i$ alleine vorkommt. Für das Altbiharium benutzen HoraceK \& LozeK (1988) das Kürzel Q1. Der Begriff Altbiharium umfasst im wesentlichen das Altpleistozän. Eine Übersicht über altpleistozäne Kleinsäugerfaunen Europas hat MAUL (1990) zusammengestellt.

FAD: Microtus (Allophaiomys) und LAD: Mimomys pusillus, Mimomys reidi

\section{Typuslokalität}

Als Typuslokalität wurde Villány 5 (H) (KRetzoi 1956, van der Meulen 1973) festgelegt (FeJfar \& HEINRICH 1990).

\section{Biostratigraphische Zuordnung}

Das Altbiharium scheint etwa gleichzeitig mit dem Altpleistozän zu beginnen und bis zur BrunhesMatuyama-Grenze zu reichen. Damit umfasst es das gesamte Altpleistozän. Eine biostratigraphische Unterteilung ist zur Zeit kaum möglich, da die Zahl der Fundstellen trotz des großen eingeschlossenen Zeitraumes noch immer relativ gering ist. In Pakefield (East Anglia) wurden in einer Fauna mit Mimomys savini und Mimomys aff. pusillus die ersten Artefakte des frühen Menschen gefunden. Die Ablagerungen sind normal magnetisiert und gehören zur Cromer Forest-bed Formation PARFITT et al. (2005). Damit reicht die Mimomys savini-Mimomys pusillus-Zone noch bis in das frühe Mittel-Pleistozän. 


\section{Zugehörige Faunen aus Mitteleuropa und angrenzenden Gebieten}

Untermaßfeld (TH), Hohensülzen (RP), Neuleiningen 11 (RP); ferner: Les Valerots (F), Couterolles (F), Chlum 6 (CZ), Stranska Skala bei Brno (CZ), Mokra 1 (CZ), Kolinany 3 (SK), Köröshegy (H), Betfia 2 (RO)

\section{Mimomys savini-Zone (Jungbiharium)}

\section{Erstbeschreibung und Definition}

Der Beginn der Mimomys savini-Zone ist durch das LAD von Mimomys pusillus, das Ende durch das FAD von Arvicola definiert worden (FEJFAR \& HEINRICH 1990).

LAD: Mimomys savini

\section{Typuslokalität}

Voigtstedt bei Sangerhausen (TH) (Georef. $11^{\circ} 19^{`} \mathrm{E}-$ 51 $\left.^{\circ} 54^{`} \mathrm{~N}\right)$, Hauptfauna (KRETzOI 1965)

\section{Unterteilung und Synonyme}

Der Begriff Mimomys savini-Faunen wurde durch Koenigswald (1973) eingeführt und später durch Koenigswald \& Heinrich (1999) ausführlich diskutiert. Er fasst die Faunen der Mimomys savini-Zone zusammen, in der Mimomys savini als einzige Art dieser Gattung auftritt. Diese Faunen gehören zum Jungbiharium. Die Mimomys savini-Faunen enden mit dem Erscheinen von Arvicola.

In der Gliederung von HoraceK \& LozeK (1988) trägt dieser Zeitabschnitt das Kürzel Q2.

\section{Zugehörige Faunen aus Mitteleuropa und angrenzenden Gebieten}

Koenigswald \& Heinrich (1999) nennen zusätzlich Kärlich B-F (RP), Süssenborn (TH), Mahlis (SN) bei Oschatz aus Deutschland sowie Prezletice (CZ), Konjeprusy C 718 bei Karlstejn (CZ), und Kozi Grziet (PL). Eine besonders wichtige Fauna dieses Faunentyps ist das Upper Freshwater Bed von West Runton in England.

\section{Cromerium s. str.}

\section{Erstbeschreibung und Definition}

Der Begriff Cromer Forst Bed wurde für fluviatile und aestuarine Sedimente an der Küste von East Anglia verwendet, die stratigraphisch teilweise in das Pliozän (Coralline Crag) und das Alt- und Mittel-Pleistozän gehören. Seit West \& WiLsON (1966) und West (1968) wird das „Cromerian” auf das „Upper Freshwater Bed” eingegrenzt. Eine revidierte Faunenliste des „Type Cromerian” gibt StUART (1975). Der Begriff Cromerium wurde von ZAGwIJN et al. (1971) erheblich erweitert. Danach schließt er mehrere Warm- und Kaltzeiten (Cromer 1-4), die vor dem Elster-Glazial liegen, ein. Biostratigraphisch gehört zumindest das Cromer 2 zu den Mimomys-savini-Faunen, während zumindest Cromer 4 zu den älteren Arvicola cantianus-Faunen gehört (Koenigswald \& KolfsChOTEN 1996).

\section{Typuslokalität}

Aestuarine Tone im Küstenprofil von West Runton in Norfolk (GB) (Georef. $\left.01^{\circ} 16^{\circ} \mathrm{E}-52^{\circ} 56^{\circ} \mathrm{N}\right)$.

\section{Biostratigraphische Zuordnung}

Das Cromerium s. str. hat eine typische Mimomys savini-Fauna geliefert (STUART 1975) und ist daher mit dem Jungbiharium im Sinne von FeJfar \& HeInRICH (1981b, 1990) gleichzusetzen.

Erweiterung des Begriffs 


\section{Zugehörige Faunen aus Mitteleuropa und angrenzenden Gebieten}

Voigtstedt (TH), Süssenborn (TH), Kärlich C, F (RP), Prezletice (CZ), West Runton (GB), usw.

\section{Toringium}

\section{Erstbeschreibung und Definition}

Das Toringium ist eine Superzone, die im mittleren Mittelpleistozän beginnt und das Holozän einschließt. Der stratigraphische Begriff wurde von Fejfar \& HeINRICH (1980) als Superzone für die Faunen aufgestellt, die durch das gemeinsame Auftreten der Gattungen Arvicola und Microtus gekennzeichnet sind. Er wurde in den Arbeiten Fejfar \& Heinrich (1981a, 1990) erläutert.

Der Beginn des Toringiums ist mit dem ersten Auftreten (FAD) der Gattung Arvicola definiert. Das Toringium schließt die Gegenwart mit ein, da Arvicola noch heute in Eurasien weit verbreitet ist.

FAD: Arvicola cantianus

\section{Typuslokalität}

Travertine aus den letzten Eem-Interglazial von Burgtonna bei Langensalza in Thüringen (Georef. $\left.10^{\circ} 44^{\circ} \mathrm{E}-51^{\circ} 04^{\circ} \mathrm{N}\right)$. Eine revidierte Faunenliste geben Koenigswald \& HeinRICH (1999).

\section{Biostratigraphische Zuordnung}

Glazialchronologisch umfasst das Toringium den jüngeren Teil des Cromer sensu latu und reicht bis zur Gegenwart, umfasst also den gesamten Bereich der drei großen nordischen Vereisungszyklen (Elster-Saale-Weichsel). Das FAD für Arvicola liegt in Mitteleuropa oberhalb der Matuyama/Brunhes Grenze aber sicherlich vor dem Elster-Glazial, also innerhalb des Mittelpleistozäns (KoENIGSwALD \& HEINRICH 1999).

\section{Unterteilung und Synonyme}

Eine weitere Möglichkeit zu Gliederung des Toringiums ergibt sich einerseits aus der Faunenzusammensetzung (Ältere und jüngere Arvicola cantianus-Faunen, sowie Arvicola terrestris-Faunen (Koenigswald 1973, Koenigswald \& Heinrich 1999) sowie die Höhe des Schmelzdickenquotienten bei Arvicola (HeINRICH 1978, 1982, 1987).

FeJfar \& Heinrich (1990) benutzen das Kürzel MQ2 für das Toringium, HoraceK \& Lozek (1988) das Kürzel Q3 und Q4. Bei Guerin $(1982,1990)$ wird dieser Zeitraum (ohne das Holozän in die Zonen MNQ22-26 unterteilt, aber die auf Nashörnern basierende Unterteilung kann in Mitteleuropa wegen des starken Faunenaustausches kaum nachvollzogen werden.

\section{Zugehörige Faunen aus Mitteleuropa und angrenzenden Gebieten}

Miesenheim 1 (RP), Kärlich G (RP), Mosbach 2 (RP), Mauer (BW), Erpfingen 1,3 (BW), Sudmer Berg 2 (NI), Noordbergum (NL), Neede (NL), Ariendorf CH (RP), Plaidter Hummerich A (RP), Schöningen II (NI), Bilzingsleben II (TH), Maastricht-Belvédère 3, 4 (NL), Hunas (BY), WeimarEhringsdorf (TH), Neumark-Nord 1 (ST), Grabschütz (SN), Stuttgart-Untertürkheim/UT (BW), Steinheim a.d. Murr (BW), Lehringen (NI), Burgtonna 1, 2 (TH), Taubach (TH), Schönfeld (BB), Gröbern (ST), Petersfels/Singen (BW), Geißenklösterle/Blaubeuren (BW), Brillenhöhle/Blaubeuren (BW), Gönnersdorf (RP), Biedensteg (HE), Ilsenhöhle/Ranis (TH), Roter Berg bei Saalfeld (TH), Rabutz bei Halle (ST), Krockstein/Rübeland (ST), Königsaue (ST), Klinge bei Cottbus (BB), Phöben bei Potsdam (BB), Meiendorf (SH), Stellmoor (SH), Pisede bei Malchin (MV). 


\section{Ältere Arvicola cantianus-Faunen}

\section{Erstbeschreibung und Definition}

Der Begriff wird von Koenigswald \& Heinrich (1999) beschreibend für einen Teilabschnitt des Toringiums für jene Säugetierfaunen Mitteleuropas benutzt, die durch Arvicola cantianus zusammen mit Sorex (Drepanosorex) savini, Talpa minor und Pliomys gekennzeichnet sind. Zur Faunenassoziation können weiterhin Megaloceros verticornis und Elephas antiquus bzw. Mammuthus trogontherii gehören. Die Faunenzone umfasst wahrscheinlich mehrere Klimaschwankungen. Sie ist jünger als die "Mimomys savini-Faunen", weil Arvicola mit ihren wurzellosen Molaren höher evoluiert ist als Mimomys savini. Eine Superposition beider Faunen ist in Kärlich (Schicht E und G) gegeben. Die “älteren Arvicola cantianus-Faunen" sind älter als die jüngeren “Arvicola cantianus-Faunen”, denen Sorex (Drepanosorex) savini, Talpa minor und Pliomys fehlen und in denen der SDQ (Schmelzdickenquotient) von Arvicola höher ist, was als Evolutionsfortschritt angesehen wird. Es gibt allerdings keine eindeutige Superposition beider Faunen. Dass diese Faunen älter als das Elster-Glazial sind, ist zwar als sehr wahrscheinlich anzunehmen, lässt sich aber bisher nirgends eindeutig belegen. Nur in Noordbergum liegt ein dem Elster zugeordneter "blauer Ton" stratigraphisch über dem Vorkommen eines Arvicola-Fundes.

FAD: Arvicola cantianus und LAD: Talpa minor, Sorex savini, Pliomys episcopalis

\section{Typuslokalität}

Koenigswald \& Heinrich (1999) haben keine Typuslokalität ausgewiesen, als solche wird hier Mie-

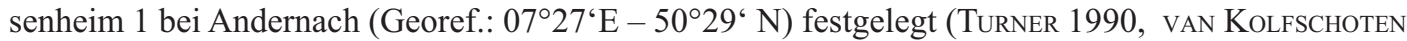
\& TURNER 1996).

\section{Biostratigraphische Zuordnung}

Mittleres Mittel-Pleistozän (im wesentlichen vor dem Elster-Glazial)

\section{Synonyme}

Diese Faunenzone wurde bei Koenigswald (1973) als “Arvicola Faunen Typ I" ausgegliedert. Diese Faunenzone entspricht dem Mosbachium, wenn man es, wie hier beschrieben, einengt und auf die Arvicola-Faunen beschränkt. Für Ungarn hat JÁNOSsY (1969) für Faunen dieser Entwicklungsstufe die Begriffe Tarkö-Phase und Vertesszöllös-Phase verwendet.

\section{Zugehörige Faunen aus Mitteleuropa und angrenzenden Gebieten}

Koenigswald \& Heinrich (1999) stellen die Faunen der Fundorte Noordbergum (NL), Miesenheim 1 bei Andernach (RP), Kärlich G im Neuwieder Becken (RP), Mosbach 2 bei Wiesbaden (RP), Mauer bei Heidelberg (BW), Erpfingen 1 und 3 bei Reutlingen (BW), Randersacker bei Würzburg (BY), Sudmer Berg 2 bei Goslar (NI) und Hundsheim bei Hainburg (A) zu den älteren Arvicola cantianus-Faunen.

\section{Mosbachium}

\section{Erstbeschreibung und Definition}

PoHLIG (1907: 79-80) bezeichnet mit dem Begriff Mosbachium einen "altdiluvialen" Zeitabschnitt, der durch das Auftreten von Elephas antiquus, Mammuthus trogontherii, Stephanorhinus etruscus, Cervus elaphus, Alces latifrons, Trogontherium sp. und Hippopotamus gekennzeichnet ist. "Es sind dies Formen, die der sogenannten Mosbacher Hauptfauna aus der mittleren Stufe des dortigen Profils zugehören" schreibt ADAM (1966: 75) und definiert damit Mosbach 2 bei Wiesbaden als Typuslokalität. Nach der damals noch unangefochtenen Vollgliederung des Quartärs nimmt ADAm (1966) an, dass das Mosbachium zeitlich an das Villafranchium anschließt und das Günz und Cromer umfasst. Er betont ausdrücklich die prä-elsterzeitliche Stellung. 


\section{Typuslokalität}

Die Typuslokalität sind die fluviatilen Sande aus dem Mündungsgebiet des Mains in den Rhein von Mosbach bei Wiesbaden (Georef. 08 16’ E - 5004' N). Da Mosbach vor 1945 ein Stadtteil von Mainz war, wird die Fundstelle auch oft als Mosbach bei Mainz geführt. Die Säugetierfauna der mehrgliedrigen Sande haben Koenigswald \& Tobien (1987) sowie Koenigswald \& Heinrich (1999) aufgelistet, wobei sie drei Faunen (Mosbach 1, 2 und 3) unterscheiden. Die von Pohlig (1907) und Adam (1966) genannten Taxa sind für das Mosbach II charakteristisch.

\section{Biostratigraphische Zuordnung}

Die Fauna des Typushorizontes (Hauptfauna von Mosbach „M2“) ist durch das Auftreten von Arvicola cantianus gekennzeichnet. Diese Form wurde durch SCHMIDTGEN (1911) mit dem jüngeren Synonym Arvicola mosbachensis bezeichnet, das auch noch benutzt wird (MAUL et al. 2000). Damit gehört Mosbach 2 zur älteren Arvicola cantianus-Fauna innerhalb des Toringiums. Wegen der wurzellosen Molaren ist Arvicola höher evoluiert als Mimomys savini und damit sind die älteren Arvicola cantianus-Faunen jünger als die Mimomys savini-Faunen, die die erste unterscheidbare Faunenzone innerhalb des Toringiums umfassen. Die Fundstellen dieser Faunen, zu denen z.B. Voigtstedt (TH), Süssenborn (TH), aber auch das klassische Upper Freshwater Bed von Cromer und West Runton (GB) gehören, enthalten kein Elephas antiquus und Hippopotamus. Nach oben ist das "prä-elsterzeitliche" Mosbachium durch den Elstervorstoß begrenzt. Die Faunen der jüngeren Arvicola cantianus-Faunen sind durch eine Faunenverarmung gekennzeichnet, die sich durch das Fehlen von Pliomys und Talpa minor deutlich macht.

Die zeitliche Umfang ist also wesentlich kürzer, als er in der Fassung von ADAm (1966) angenommen wurde und bildet die zweite Phase des Mittelpleistozäns.

\section{Synonyme}

Nach der Zusammensetzung der Fauna entspricht das Mosbachium den Arvicola-Faunen Typ 1 bei Koenigswald (1973) bzw. den älteren Arvicola cantianus-Faunen bei Koenigswald \& HeinRich (1999). FeJFAR (1976) hat den Namen Mauer-Stufe vorgeschlagen.

\section{Zugehörige Faunen aus Mitteleuropa und angrenzenden Gebieten}

Koenigswald \& Heinrich (1999) stellen zu den älteren Arvicola cantianus-Faunen die Fundorte Erpfingen 2 und 3 (BW), Mauer (BW), Miesenheim 1 (RP), Sudmer Berg 2 (NI), wahrscheinlich auch Jockgrim (RP) und Würzburg Schalksberg (BY), ferner Hundsheim (A) und Tarkö (H).

\section{Jüngere Arvicola cantianus-Faunen}

\section{Erstbeschreibung und Definition}

Der Begriff wird von KoEnigswald \& HeInRICH (1999) beschreibend für einen Teilabschnitt des Toringiums für jene Säugetierfaunen Mitteleuropas benutzt, die durch Arvicola cantianus gekennzeichnet sind, denen aber Sorex (Drepanosorex) savini, Talpa minor und Pliomys unter den Kleinsäugern und Megaloceros verticornis bei den Großsäugern fehlen. Der SDI (Schmelzdickenquotient) von Arvicola ist kleiner als der in den "älteren Arvicola cantianus-Faunen" was als Evolutionsfortschritt angesehen wird.

Diese Faunenstufe wird jünger als die "älterem Arvicola-cantianus Faunen" angesehen und gehen nach oben in die Arvicola terrestris-Faunen über, wobei sich eine Übergangszone mit Arvicola cantianus/terrestris dazwischen schaltet.

LAD: Arvicola cantianus

\section{Typuslokalität}

KoEnigswald \& HeinRich (1999) haben keine Typuslokalität ausgewiesen, weil die Faunenzone mehrere Klimaschwankungen umfasst. Als Typuslokalität benennen wir hier Bilzingsleben II (Georef. $11^{\circ} 04^{\circ}$ E $-51^{\circ} 17^{`} \mathrm{~N}$ ), dessen Fauna Mania et al. (1997) und HeInRICH (1997) zusammengefasst haben. 


\section{Synonyme}

Diese Faunenzone wurde bei Koenigswald (1973) als Arvicola-Faunen Typ 2 ausgegliedert.

\section{Biostratigraphische Zuordnung}

Jüngeres Mittel-Pleistozän. Die jüngeren Arvicola cantianus-Faunen liegen nach derzeitigen Vorstellungen zeitlich über dem Elster-Glazial und reichen mit den "Arvicola cantianus/terrestris-Faunen" bis in das letzte Interglazial, das Eem.

\section{Zugehörige Faunen aus Mitteleuropa und angrenzenden Gebieten}

Folgende Faunenfundorte gehören zu den "jüngeren Arvicola cantianus-Faunen": Wageningen-Franse Kamp (NL), Neede (NL), Ariendorf bei Linz (RP), mehrere Fundpunkte aus dem Neuwieder Becken (Plaidter Hummerich, Schweinskopf-Karmelenberg, Wannenköpfe und Tönnchesberg 1) (RP), Stuttgart Bad Cannstatt (BW), Steinheim an der Murr (BW), Schöningen II (NI), Bilzingsleben II (TH) und Schönfeld bei Cottbus (BB).

\section{Arvicola terrestris-Faunen}

\section{Erstbeschreibung und Definition}

Der Begriff wird von KoEnigswald \& HeInRICH (1999) beschreibend benutzt, aber nicht ausdrücklich definiert. Eine Definition wird hier gegeben. Die Arvicola terrestris-Faunen folgen auf die jüngeren Arvicola cantianus-Faunen. Es kann sich eine Übergangszone, die Arvicola cantianus-terrestris Zone einschieben. Gekennzeichnet sind die Arvicola terrestris-Faunen durch A. terrestris, die sich durch eine SDQ deutlich unter 100 als höher evoluiert als A. cantianus erweist. Die Arvicola terrestrisFaunen umfassen drei stratigraphische Niveaus, die in Mitteleuropa ökologisch sich sehr stark unterscheiden, nämlich der jüngere Teil des letzten Interglazials, das gesamte Weichsel-Glazial und das Holozän. Trotz der starken ökologischen Veränderungen ist die kennzeichnende Art, wenn auch mit erheblichen Größenunterschieden, vertreten. Die biostratigraphische Gliederung dieses Zeitabschnittes ist bei KoEnigswald \& HeInRICH (1996) ausführlich dargestellt.

FAD: Arvicola terrestris

\section{Typuslokalität}

Weinberghöhlen bei Neuburg/Donau (BY) (Georef. $\left.11^{\circ} 03^{\circ} \mathrm{E}-48^{\circ} 46^{\circ} \mathrm{N}\right)$ Schicht $\mathrm{C}$ mit einer reichen Fauna aus dem Gravetian (Koenigswald et al. 1974, Koenigswald \& Müller-Beck 1975)

\section{Biostratigraphische Zuordnung}

Jung-Pleistozän mit Eem und Weichselglazial wie Holozän.

\section{Synonyme und Unterteilungen}

Ökologisch lassen sich das Eem, das Weichsel und das Holozän unterscheiden. Das Holozän ist durch eine starke Reduktion der Biodiversität gekennzeichnet. Eine Minderung in der Artenvielfalt lässt sich schon seit dem Mittelpleistozän mit zunehmender Intensität beobachten.

GuERIN $(1982,1990)$ ordnet seine Faunenzone MNQ25 dem Eem zu, und MNQ26 dem Weichsel. Das Holozän bleibt aber unberücksichtigt. HorAceK \& LozeK (1988) weisen das Weichsel-Glazial und das Holozän als Q4 aus, geben aber keine klare Abgrenzung zu ihrem Q3, das das Mosbachium umschreibt.

\section{Zugehörige Faunen aus Mitteleuropa und angrenzenden Gebieten}

Über die gut untersuchten Arvicola terrestris-Faunen aus dem Jungpleistozän (Eem, Weichsel) und dem Holozän liegt ein so umfangreiches Schrifttum vor, dass hier auf eine Auflistung verzichtet und auf die Zusammenstellungen bei Koenigswald \& Heinrich $(1996,1999)$ und Koenigswald (2002) verwiesen werden darf. 


\section{Steinheimium}

\section{Erstbeschreibung und Definition}

KRETZOI (1969) benutzt den Begriff "Steinheimian" als biostratigraphische Einheit für die Faunen mit Elephas antiquus und Stephanorhinus kirchbergensis, also für Arvicola-Faunen des Mittelpleistozäns.

\section{Typuslokalität}

Interglaziale Flussablagerungen (Antiquus-Schotter) in Steinheim an der Murr bei Marbach (BW) (Georef: $09^{\circ} 17^{`} \mathrm{E}-48^{\circ} 58^{\circ} \mathrm{N}$ ). Die Fauna wurde von ADAm (1954) eingehend beschrieben und ist bei KoEnigswald \& HeInRICh (1999) aufgelistet.

\section{Biostratigraphische Zuordnung}

FEJFAR (1976) spezifiziert den von KRETzOI (1969) eingeführten Begriff und unterteilt ihn in eine untere Phase Mauer und eine obere Phase Swanscombe, die mit den Glazialen und Interglazialen der damaligen Vorstellung korreliert. Danach endet sein Steinheimium innerhalb des Saale. Diese Obergrenze ist allerdings kaum fassbar. Später wurde auch die Taubach-Phase (Eem) in das Steinheimium mit einbezogen (Fejfar \& Heinrich 1983). Savage \& Russel (1983) fassen unter dem Begriff Steinheimian die Faunen zwischen 400000 und 5000 Jahren v.h. zusammen. Die Untergrenze entspricht damit etwa der von KRETZOI (1969) und FeJFAR (1976) benutzten Gliederung, die Abgrenzung gegenüber der rezenten Fauna (5000 a) hat keinerlei biostratigraphischen Hintergrund.

Das Steinheimium liegt vollständig innerhalb des Toringium, bildet aber keine besonders sinnvolle Abgrenzung. Dieser Zeitraum wird besser durch die jüngeren Arvicola cantianus-Faunen und Arvicola terrestris-Faunen beschrieben.

\section{Zugehörige Faunen aus Mitteleuropa und angrenzenden Gebieten}

Wegen der Unschärfe des stratigraphischen Begriffes und der Überschneidung mit den wesentlich besser definierten Arvicola-Faunen wird hier auf eine Auflistung von Fundstätten verzichtet.

\section{Holozän}

Die holozäne Fauna Mitteleuropas wurde biostratigraphisch nie von den älteren Faunen begrifflich abgegrenzt, zum einen weil zur Kennzeichnung die Bezeichnung „rezente Fauna” im allgemeinen ausreicht, und zum anderen weil sie kaum Gegenstand paläontologischer Forschung ist. Nach den biostratigraphischen Charakteristika gehört sie eindeutig zum Toringium, speziell zu den Arvicola terrestris-Faunen. Der Unterschied zu den pleistozänen Arvicola terrestris-Faunen liegt bei der endemischen Fauna in dem betont warmzeitlichen (interglazialen) Faunenbestand. Gegenüber dem Eem fehlen allerdings einige wichtige Großsäuger wie Elefanten und Nashörner und klimatisch besonders anspruchsvolle Faunenelemente wie Hippopotamus und Bubalus. In historischer Zeit ist der Artbestand durch den Menschen erheblich verändert worden, sei es durch das Ausrotten zahlreicher Arten, besonders bei den Raubtieren, aber auch durch die Einbürgerung exotischer Formen (KoENIGSWALD 2002). Regional könnte man daher durchaus eine biostratigraphische Feingliederung entwickeln, wie sie in Ansätzen von KRETzor (1969) für das Karpatenbecken vorgelegt wurde. 


\section{Abkürzungen}

Für die europäischen Staaten bzw. die deutschen Bundesländer werden die folgenden Kürzel verwendet:
$\mathrm{A}=$ Österreich
$\mathrm{BB}=$ Brandenburg
$\mathrm{BW}=$ Baden-Württemberg
$\mathrm{BY}=$ Bayern
$\mathrm{CZ}=$ Tschechische Republik
$\mathrm{E}=$ Spanien
$\mathrm{F}=$ Frankreich
$\mathrm{GB}=$ Großbritannien
$\mathrm{GR}=$ Griechenland
$\mathrm{H}=$ Ungarn.
$\mathrm{HE}=$ Hessen
$\mathrm{I}=$ Italien

MV $=$ Mecklenburg-Vorpommern

$\mathrm{NI}=$ Niedersachsen

$\mathrm{NL}=$ Niederlande

$\mathrm{NW}=$ Nordrhein-Westfalen

$\mathrm{PL}=$ Polen

$\mathrm{RO}=$ Rumänien

$\mathrm{RP}=$ Rheinland-Pfalz

$\mathrm{SH}=$ Schleswig-Holstein

SK = Slowakische Republik

$\mathrm{SN}=$ Sachsen

$\mathrm{ST}=$ Sachsen-Anhalt

$\mathrm{TH}=$ Thüringen 